Article

\title{
The Over-Expression of E2F3 Might Serve as Prognostic Marker for Neuroblastoma Patients with Stage 4S Disease
}

\author{
Stefano Parodi ${ }^{1, *(D)}$, Marzia Ognibene ${ }^{2}$ (D) Riccardo Haupt ${ }^{1}$ and Annalisa Pezzolo ${ }^{2, *(D)}$ \\ 1 U.O. Epidemiologia e Biostatistica, IRCCS Istituto Giannina Gaslini, 16147 Genova, Italy; \\ riccardohaupt@gaslini.org \\ 2 U.O.C. Laboratorio Cellule Staminali Post Natali e Terapie Cellulari, IRCCS Istituto Giannina Gaslini, \\ 16147 Genova, Italy; marziaognibene@gaslini.org \\ * Correspondence: stefanoparodi@gaslini.org (S.P.); annalisapezzolo56@gmail.com (A.P.); \\ Tel.: +39-010-56363531 (S.P.); Fax: +39-010-3779820 (A.P.)
}

Received: 20 April 2020; Accepted: 14 May 2020; Published: 16 May 2020

\begin{abstract}
Stage 4S neuroblastoma is a childhood cancer occurring in infants ( $<12$ months at diagnosis) with metastases limited to liver, skin, and bone marrow $(<10 \%)$. It is associated with an excellent outcome, due to its notable ability to undergo spontaneous regression without any therapeutic intervention. However, a subgroup of patients is doomed to relapse and eventually to die in spite of aggressive therapies. Stage $4 S$ neuroblastoma shows characteristic hypermethylation of genes involved in the telomere maintenance, indicating that the dysregulation of these genes might serve as prognostic marker. The retinoblastoma tumor suppressor protein (RB)-E2F transcription factors pathway is one of the critical tumor-suppressor/oncogene pathways involved in regulating telomerase expression. We have interrogated in silicopublic neuroblastoma databases for regulators involved in the RB-E2F pathway especially for E2F factors themselves, and we identified the E2F transcription factor 3 (E2F3) expression as a potential prognostic marker in stage $4 S$ neuroblastoma. In order to confirm this finding, we screened 38 paraffin-embedded tissue samples stage $4 \mathrm{~S}$ neuroblastoma for E2F3 protein expression using immunofluorescence, and we observed that augmented expression was strongly associated with impaired event-free survival. These results indicate that E2F3 expression might serve as prognostic marker in patients with stage $4 S$ disease.
\end{abstract}

Keywords: neuroblastoma; stage 4S disease; E2F3 protein; prognostic marker

\section{Introduction}

Neuroblastoma (NB) is the most common extra-cranial solid tumor of childhood, and it is the main cause of death in children between 1 and 4 years [1,2]. NB is considered a developmental disorder resulting from the interruption of normal sympathetic neuronal progenitor maturation [3]. NB is a heterogeneous disease with prognosis ranging from long-term survival to fatal outcome [4]. The clinical and biological parameters are used for therapeutic stratification of NB patients $[5,6]$. Stage 4S NB is a special type of NB established in infants with metastases limited to the liver, skin, and bone marrow ( $<10 \%)$ at diagnosis and is associated with an excellent outcome due to its notable ability to undergo spontaneous regression without any therapeutic intervention [7]. Unfortunately, a subgroup of patients with stage $4 \mathrm{~S}$ disease has worse outcomes. Prognostic factors at diagnosis include MYCN amplification, recurrent segmental chromosomal aberrations (losses of chromosome arms 1p, 3p, 4p, 6q, 11q and gains of chromosome arms 1q, 2p, 17q), diploid DNA index, age younger than 4 weeks, and life-threatening symptoms [8-12]. Thus, it is imperative to identify new therapeutic targets and to establish differentiation inducing protocols. The exact mechanisms responsible for 
spontaneous regression or differentiation into a benign ganglioneuroma without treatment are unknown. Several possible mechanisms have been proposed to explain spontaneous regression: neurotrophin deprivation, loss of telomerase activity, cellular or humoral immunity, and alterations in epigenetic regulation [13-15]. It has been demonstrated that the DNA methylation pattern of stage 4S NB is characterized by differential methylation of target genes of transcription factors involved in neural crest development and neuronal differentiation $[13,14]$. The DNA methylation portrait is anew mechanism that may contribute to the stage $4 \mathrm{~S}$ tumor progression or spontaneous regression. The telomerase reverse transcriptase (TERT) gene encoding the catalytic subunit of telomerase involved in telomere length regulation [15] and its promoter [16] are hypermethylated in NB samples from patients with stage 4S disease [13]. Interestingly, stage $4 S$ tumors showed characteristic hypermethylation of genes involving in an important pathway the retinoblastoma tumor suppressor protein (RB)-E2F oncogene transcription factors [17]. The RB-E2F pathway is one of the critical tumor-suppressor/oncogene pathways involved in regulating TERT gene expression [17]. It has been demonstrated that TERT is expressed at lower levels in stage $4 \mathrm{~S}$ compared to stage 4 NB and that low TERT activity or short telomeres might be associated with spontaneous regression of this special type of NB [14,18]. The retinoblastoma tumor suppressorRB1 gene encodes for a protein $\mathrm{pRB}$ that acts as a tumor suppressor regulating cell growth and keeps cells from dividing too fast or without control [19]. Inactivation of RB1 expression in tumor cells leads to the deregulation of activity of transcription factors E2F1, E2F2, and E2F3, which control the expression of genes involved in differentiation, development, proliferation, and apoptosis [20-24]. To notice, the transcription factors E2F1, E2F2, and E2F3 bind to the proximal MYCN promoter, specifically in NB cell lines expressing MYCN [21]. Several transcription factors that are clue in normal neuronal development, as well as the cell cycle regulator E2F3, were found to be up-regulated in a murine model of human MYCN-driven NB [22]. E2F3 is part of the E2F family of transcription factors that includes eight members (E2F1-8) [20]. It has been suggested that miR-34a could have a role as tumor suppressor in NB tumorigenesis by directly binding to E2F3 mRNA and significantly reducingthe level of E2F3 protein [25]. However, no studies have examined the role of RB-E2F pathway in stage $4 \mathrm{~S}$ NB. The expression of $R B 1$ gene may be responsible for the block of cell cycle progression and decreased TERT activity in stage $4 S$ undergoing spontaneous regression. A crucial goal should be to determine whether the over-expression of one or more of the genes involved in RB-E2F pathway and of TERT gene might serve as prognostic markers for patients with stage $4 S$ with worse outcomes. Here, we have examined in silicothree public NB databases from R2 platform for RB1, E2F1, E2F2, E2F3, and TERT gene expressions.

\section{Results}

\subsection{Association of RB1, TERT, E2F1, E2F2, and E2F3 Gene Expressionswith Clinical Outcome in Stage 4S Neuroblastoma Patients}

We examined how RB1, TERT, E2F1,E2F2, and E2F3 gene expressions linked to event-free survival (EFS) in stage 4S NB patients using gene expressions in the publicly available datasets consisting of primary tumor samples from three independent NB patient cohorts (Kocak-649 [26], Oberthuer-251 [27], and SEQC-RPM [28] datasets), downloaded from the R2: Genomic Analysis and Visualization Platform (available online: http://r2.amc.nl). The three data sets included microarray expression profiles of 134 stage 4S NB with MYCN normal status and 32 events, defined as disease relapse, disease progression, or death for any cause. In more details, Kocak-649 included 78 stage $4 S$ NB patients, of which 17 were excluded for lack of information on patients' survival, 4 for MYCN amplification, and 1 for missing data about MYCN status, thus leaving 56 patients (13 events) available for the analyses. Oberthuer-251 included 31 patients and among these one with $M Y C N$ amplification; then, the analyses were carried out on 30 patients (7 events). Finally, SEQC-RPM, included 53 patients, of which 5 were excluded for MYCN amplification $(n=4)$ or missing data for MYCN status $(n=1)$, leaving 48 patients available for the analyses (12 events). The SEQC-custom data set, which contains information from the same samples of SEQC-RPM, profiled by a different microarray platform, was analyzed separately. For 
each gene, patients were split into two groups of the same size, using the median value of gene expression. Sensitivity analysis was carried out by splitting the patients on the basis of two different cut-offs (the first and the last tertile of the expression values distribution). Figure 1A-E shows the results of EFS analysis as a forest plot, obtained displaying the hazard ratio (HR) estimates for each gene, the corresponding meta-analytic HR estimate (mHR), and their related 95\% Confidence Intervals (95\% CI). The corresponding values are reported in Table 1.

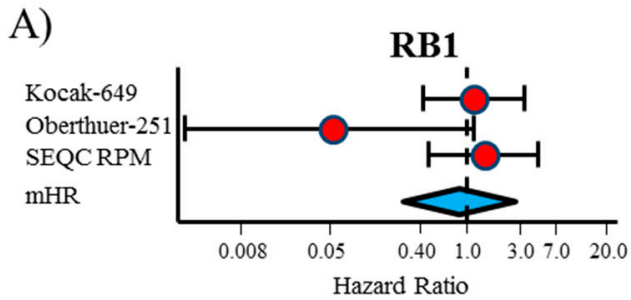

B)

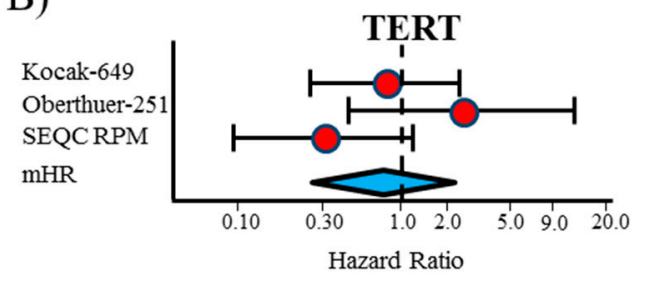

C)

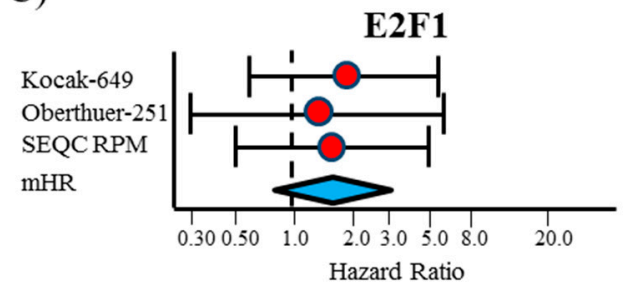

D)

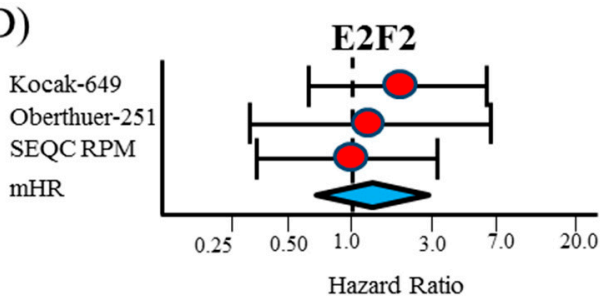

E)

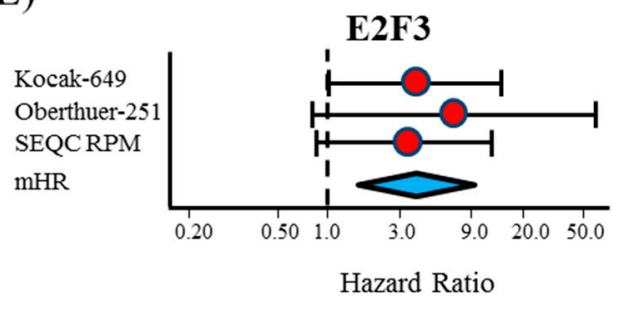

Figure 1. Forest plot of hazard ratios for the association between Event Free Survival of patients with Stage $4 S$ neuroblastoma and the considered gene expressions, categorized on the basis of the median expression value. (A) RB1; (B) TERT; (C) E2F1; (D) E2F2; (E) E2F3. mHR = meta-analytic estimate of HR.

Table 1. Hazard ratios for the association between event free survival of patients with Stage 4 S neuroblastoma and the considered gene expressions, categorized on the basis of the median expression value.

\begin{tabular}{ccclc}
\hline Data Set & N/E & HR & $\mathbf{9 5 \%}$ CI & $p$ \\
\hline RB1 & & & & \\
Kocak-649 & $56 / 13$ & 1.2 & $0.39-3.4$ & 0.795 \\
Oberthuer-251 & $30 / 7$ & 0.06 & $0.00-0.47$ & 0.003 \\
SEQC-RPM & $48 / 12$ & 1.4 & $0.45-4.5$ & 0.546 \\
mHR & $134 / 32$ & 0.87 & $0.27-2.8$ & 0.821 \\
TERT & & & & \\
Kocak-649 & $56 / 13$ & 0.82 & $0.27-2.4$ & 0.719 \\
Oberthuer-251 & $30 / 7$ & 2.5 & $0.48-12.7$ & 0.266 \\
SEQC-RPM & $48 / 12$ & 0.33 & $0.09-1.2$ & 0.082 \\
mHR & $134 / 32$ & 0.80 & $0.29-2.2$ & 0.666 \\
E2F1 & & & & \\
Kocak-649 & $56 / 13$ & 1.9 & $0.61-5.7$ & 0.268 \\
Oberthuer-251 & $30 / 7$ & 1.3 & $0.30-6.0$ & 0.705 \\
SEQC-RPM & $48 / 12$ & 1.6 & $0.50-5.0$ & 0.429 \\
mHR & $134 / 32$ & 1.6 & $0.80-3.3$ & 0.177 \\
\hline
\end{tabular}


Table 1. Cont.

\begin{tabular}{ccccc}
\hline Data Set & N/E & HR & 95\% CI & $p$ \\
\hline E2F2 & & & & \\
Kocak-649 & $56 / 13$ & 2.0 & $0.64-6.0$ & 0.233 \\
Oberthuer-251 & $30 / 7$ & 1.4 & $0.31-6.1$ & 0.675 \\
SEQC-RPM & $48 / 12$ & 1.0 & $0.34-3.2$ & 0.942 \\
mHR & $134 / 32$ & 1.4 & $0.70-2.9$ & 0.330 \\
E2F3 & & & & \\
Kocak-649 & $56 / 13$ & 3.8 & $1.0-13.7$ & 0.031 \\
Oberthuer-251 & $30 / 7$ & 6.8 & $0.81-56.2$ & 0.040 \\
SEQC-RPM & $48 / 12$ & 3.3 & $0.89-12.2$ & 0.058 \\
mHR & $134 / 32$ & 3.9 & $1.7-9.1$ & 0.002 \\
\hline
\end{tabular}

$\mathrm{N} / \mathrm{E}=$ number of samples/number of events; $\mathrm{HR}=$ Hazard Ratio; $95 \% \mathrm{CI}=95 \%$ Confidence Intervals of HR; $\mathrm{mHR}=$ meta-analytic estimate of HR.

For RB1 gene expression, a large heterogeneity was observed between the three considered data sets (Figure 1A). The corresponding mHR was close to one (0.87, 95\% CI: 0.27-2.8), the expected value under the null hypothesis of no association between gene expression and patient's survival (Table 1). The corresponding Kaplan-Meier curves revealed a noticeable heterogeneity, with a poor survival rate in patients with low RB1 expression in the Oberthuer data set, but not in the other databases (Figure S1). A similar pattern also emerged in the sensitivity analysis at two different cut-offs (Figures S2-S4).

No association was observed between TERT gene expression and EFS (mHR $=0.80,95 \% \mathrm{CI}$ : 0.29-2.2, Figure $1 \mathrm{~B}$ and Table 1). Analysis at each selected cut-off did not point out any prognostic role of TERT expression (Figures S5-S8), even if in the SEQC-RPM data set a poorer survival was observed for patients with expression values lower than the median (Figure S5C) and the first tertile (Figure S6C), but without achieving statistical significance.

A low E2F1 expression was slightly associated with a better EFS in the three considered data sets and, accordingly, in the meta-analytic analysis, even if statistical significance was not reached $(\mathrm{mHR}=1.6,95 \% \mathrm{CI}: 0.80-3.3$, Figure 1C and Table 1). A higher EFS was consistently observed for patients with low E2F1 gene expression in each considered data set and for each selected cut-off (Figures S9-S11), which was statistically significant when patients were categorized on the basis of the first tertile $(\mathrm{mHR}=3.0,95 \% \mathrm{CI}: 1.2-7.8$, Figure S12A).

High E2F2 expression was slightly associated with a poor EFS in all considered data sets, except SEQC-RPM (Figure 1D and Figure S13), but statistical significance was not reached ( $\mathrm{mHR}=1.4,95 \% \mathrm{CI}$ : $0.70-2.9$, Table 1). In the sensitivity analysis (Figures S14-S16), this association seemed to be slightly stronger when the first tertile of the gene distribution was selected as cut-off.

Finally, high levels of E2F3 gene expression were associated to a poor survival in each considered data set (Figure 1E, and Figure 2A-C). The corresponding mHR was 3.9 (95\% CI: 1.7-9.1, $p=0.002$, Table 1). Results from sensitivity analysis consistently confirmed the association observed at each selected cut-off (Figures S17-S20).

The role of E2F3 in the prognosis of stage 4S NB patients with $M Y C N$ amplification is worthy of further investigations. Unfortunately, in the data sets analyzed stage 4S NB patients with tumor MYCN amplified are only 9 (4 in Kocak-649, 1 in Oberthuer-251, and 4 in SEQC-RPM database respectively), thus preventing survival analysis from being performed. 
A)

B)

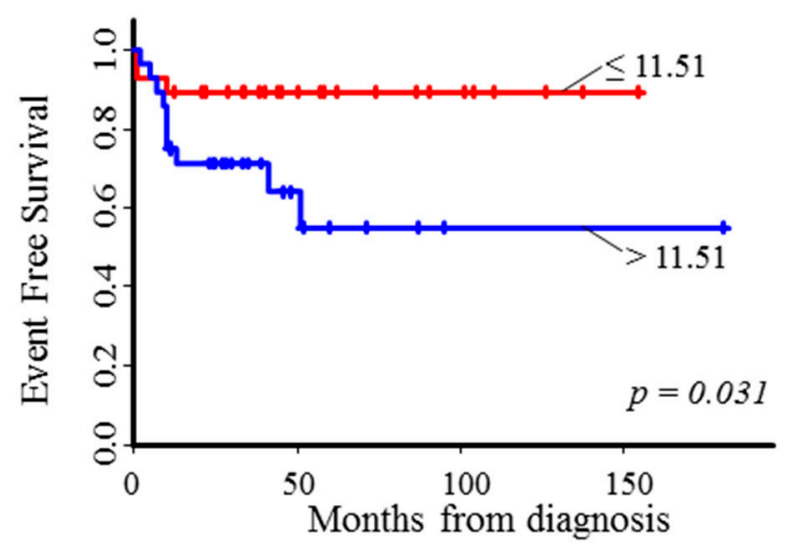

C)
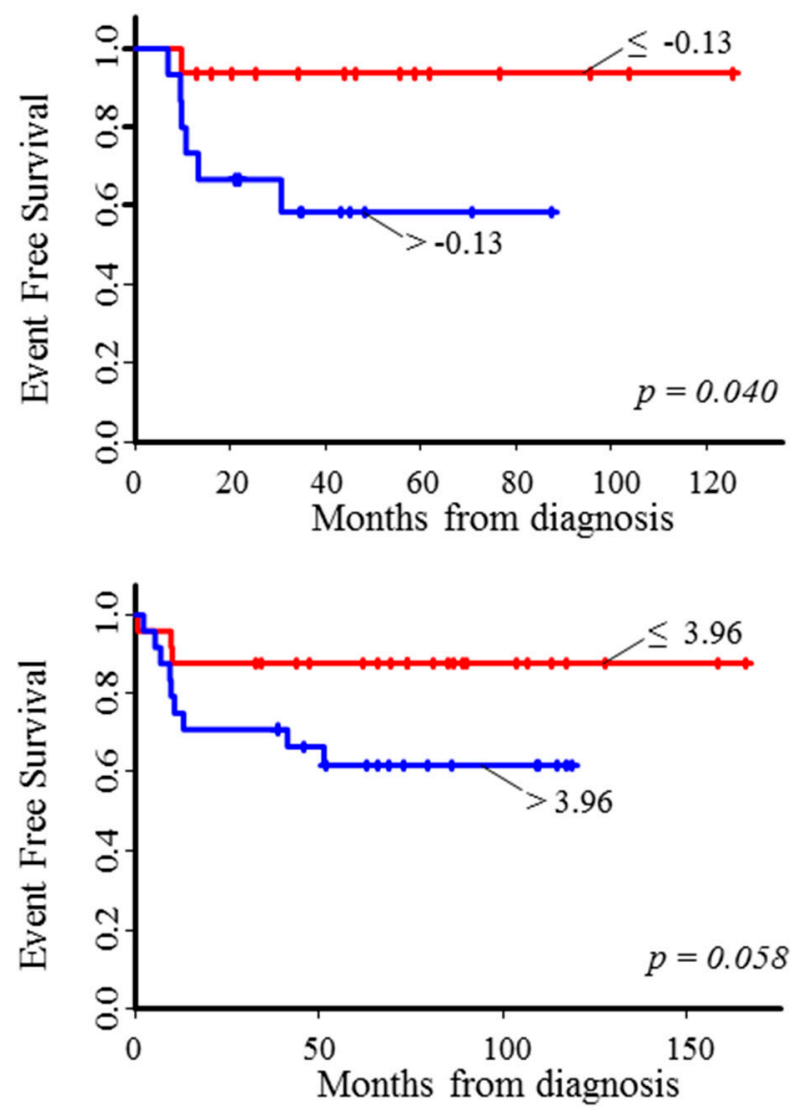

Figure 2. Event Free Survival curves of stage $4 S$ neuroblastoma patients in relation to E2F3 gene expression levels in the three considered microarray datasets. (A) Kocak-649; (B) Oberthuer-251; (C) SEQC-RPM. Patients were split into two groups (red line: lower values; blue line: higher values) on the basis of the median value of gene expression (reported near the corresponding curve).

\subsection{E2F3 Protein Expression in Primary Stage 4 S Neuroblastoma Tissue Sections}

NB paraffin-embedded tissue sections of NB tumors from 38 patients with stage $4 \mathrm{~S}$ disease were tested for E2F3 protein expression by immunofluorescence. Twenty-four patients did not have relapse or tumor progression after a median follow up time of 2.9 months (range: 9 days-18.3 months). One patient relapsed at a local site, and 13 experienced distant metastases. These included 4 stage 4 sites (namely: 1 lung, 1 central nervous system, and 2 bones, who also included bone marrow in both and liver in one), 3 distant and local sites combined, and 6 metastatic sites only. Median follow up time 
among non-relapsed patients was 109 months (range: 43.3-187.1 months). All patients, but 5 (3 non relapsed and 2 relapsed), had a normal $M Y C N$ status.

Brilliant and intense green nuclear staining for E2F3 was present in the specimens from relapsed patients indicative of strong expression. Weak green nuclear staining was detected in the samples from not relapsed patients indicative of low expression of E2F3 protein. The number of cells with intense nuclear staining for E2F3 protein was higher in tissues from primary stage 4S tumors that relapsed or progressed compared to not relapsed tumors showing weak intensity of E2F3 nuclear staining ( $p<0.001$, chi squared test) (Figure 3A,B and Table S1). For control, the proportion of cells with nuclear staining for E2F3 protein was higher in tissues from primary stage 4S NB than in normal adjacent tissues (E2F3 ${ }^{+}$nuclei $90 \pm 3 \%$ vs. $10 \pm 1 \%, p=0.002$ ) (Figure 3C).

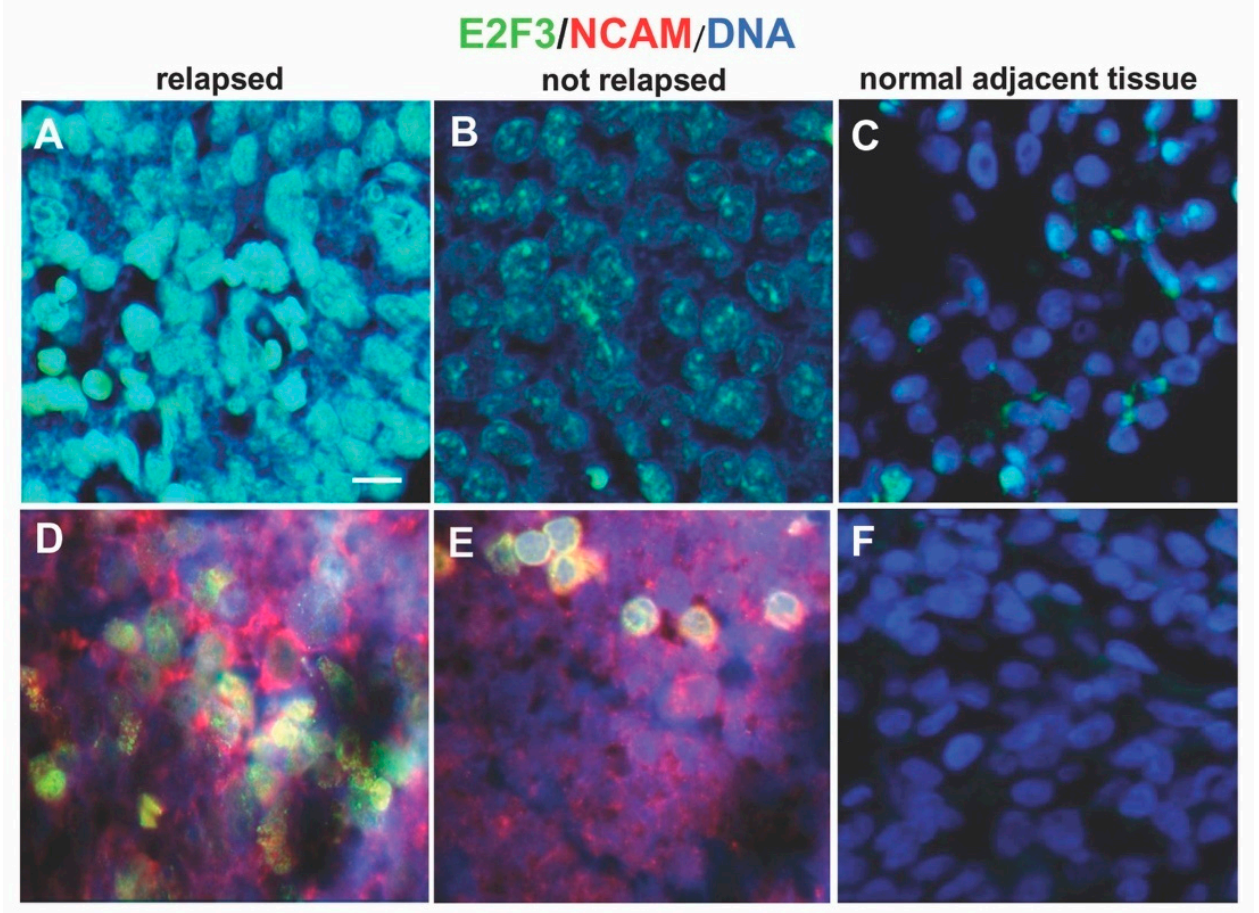

Figure 3. Expression of E2F3 protein. Immunofluorescence assay was performed to test the expression of E2F3 protein in neuroblastoma $4 \mathrm{~S}$ tissues using the anti-E2F3 antibody. (A) Representative image showing brilliant green nuclear staining for E2F3 present in one specimen out of the 14 relapsed patients analyzed. (B) Representative image presenting weak green nuclear staining of E2F3 detected in one sample from not relapsed patients. (C) E2F3 protein expression in normal adjacent tissues. (D,E) Representative images of tumor cells (red) identified using the NB specific marker NCAM (CD56). (F) Normal adjacent tissues negative for NCAM. DAPI was used for DNA staining and is shown as blue. Three independent experiments were performed. Scale bars: $25 \mu \mathrm{m}$.

\section{Discussion}

Patients with stage 4 S disease $(7 \%-10 \%$ of NB) generally have favorable outcomes, often exhibiting spontaneous maturation and regression, or needing moderate intensity chemotherapy, or low dose of radiotherapy to the liver [1-6]. Unfortunately, $10 \%-25 \%$ of patients with stage 4 S NB do not survive, mostly due to either rapid progression of liver metastases, or development of hepatomegaly related to disease-causing compression of normal liver tissue, lungs, kidneys, inferior vena cava, and intestines [7-10]. Tumor progression to high risk stage 4 is observed occasionally [8]. Disease progression of stage $4 S$ is strongly related to the presence of unfavorable prognostic biologic markers in the tumor and age younger than 4 weeks at diagnosis [8-12]. Stage $4 S$ NB represents two biologically different sub-groups, one of which shows whole-chromosome numerical abnormalities typical of localized tumors (stages 1,2,3), whereas the other carries recurrent imbalance chromosomal 
structural rearrangements, as well as TERT activity, representative of clinically advanced tumors (stage 4) [29]. Notably, it has been suggested that stage $4 \mathrm{~S}$ spontaneous regression is related to the loss of TERT activity and telomere shortening [14,18]. Our group examined the telomere length, TERT expression, and the presence of alternative lengthening of telomeres (ALT) mechanism in $100 \mathrm{NB}$ samples; most of the $4 \mathrm{~S}$ specimens had low TERT expression or short telomeres associated with a good prognosis [18]. A recent study found that NB patients whose tumors lacked telomere maintenance had an excellent prognosis, whereas patients whose tumors harbored TERT gene over-expression had worse outcome [30]. The aggressive stage 4 NB expressed high levels of TERT, whereas tumors with favorable outcome had no or little expression [30]. Spontaneous tumor regression without treatment occurred in $4 \mathrm{~S} \mathrm{NB}$ patients with no telomere maintenance [30]. It is likely that a better understanding of the mechanism(s) of tumor progression or spontaneous regression/differentiation will support the identification of targeted therapeutic approaches for stage 4S NB patients.

The RB-E2F axis is one of the critical tumor-suppressor/oncogene pathways involved in regulating $T E R T$ expression. Inactivation of $R B 1$ expression in cancer leads to the deregulated activity of the transcription of E2F1, E2F2, and E2F3 [24].

Here we examined how RB1,E2F1,E2F2, E2F3, and TERT gene expressions linked to survival in stage $4 \mathrm{~S}$ NB patients using microarray mRNA expression data from three independent public NB patient cohorts. We have identified that RB1 and TERT gene expressions had no association with EFS; low E2F1,E2F2 expression was slightly associated with a good EFS in all considered data sets, whereas high levels of $E 2 F 3$ gene expression were associated to a poor survival in each considered data set. E2F3 could be a new potential prognostic marker of stage $4 \mathrm{~S}$ NB without $M Y C N$ amplification. In the data sets analyzed in this work, only 9 stage $4 \mathrm{~S}$ NB patients presented $M Y C N$ amplified tumor; however, we could not analyze either the E2F3 expression or its relation with survival of this group of patients.

$\mathrm{E} 2 \mathrm{~F} 3$ is the major member of the E2F family protein and has an important role in regulating gene transcription, cell cycle, proliferation, and apoptosis [31,32]. It has been described that E2F3 protein regulated $M Y C N$ transcription and is required for full activity of the MYCN promoter in NB [21]. The up-regulation of the transcription factor encoded by the $M Y C N$ gene is essential for cell-cycle control, growth-factor dependence, apoptosis, and block of differentiation in primary neural crest cells [22]. Over-expression of E2F3 protein that accompanies activation of the MYCN gene might represent the driving factor of stage $4 S \mathrm{NB}$ progression.

It has been shown that elevated level of E2F3 protein drives ectopic proliferation in multiple tissues [33]. Interestingly, E2F3 is an oncogene with strong proliferative potential, and it is over-expressed in multiple cancers [34-37]. Amplification or elevated expression of the E2F3 locus at 6p22.3 has been identified in breast [34], prostate [35], bladder [36], and lung cancers [37]. Gain or amplification of chromosome 6p22.3 is not recurrent in NB [2,4,38], and E2F3 gene expression is not activated bona fide through chromosomal abnormalities, therefore an oncogenic mechanism probably exists that operates independently of gene amplification. We postulate that still-unknown factors affect the level and timing of oncogene over-expression and determine the specific mechanism used by neuroblast to aberrantly express each oncogene. Moreover, cancer genome-wide association studies (GWAS) showed that the malignant transformation of developing neuroblast is influenced by common variations (polymorphisms) in the genome at 6p22.3 and that the NB phenotype is in part determined by germline variations at this locus $[39,40]$. These data suggest that the chromosome 6p22.3 risk alleles not only influence the developing NB but also the likelihood ofdeveloping a more malignant phenotype $[39,40]$. It is not known how the presence of common DNA variants at 6p22.3 locus contributes to the risk of neuroblastic malignant transformation, presumably through altered expression and/or alternative splicing of regional candidate genes. Here, we reported that the E2F3 gene located at $6 \mathrm{p} 22.3$ appeared to play an oncogenic role in NB 4S stage. Using publicly available mRNA expression data, we have demonstrated that E2F3 high expression is associated with reduced survival of NB patients with stage $4 \mathrm{~S}$ disease. For further confirmation, we performed a retrospective investigation detecting a high level of nuclear staining for $\mathrm{E} 2 \mathrm{~F} 3$ protein in $4 \mathrm{~S}$ tumors with the occurrence 
of relapse or progression, whereas the non-progressing tumors had low level of this protein, suggesting that E2F3 over-expression may represent an earlier event in NB progression. Genomic studies have demonstrated that tumors that amplify E2F3 often also acquire mutations in RB1 with consequent inactivation of the RB pathway [41], but we did not find any association between $R B 1$ gene expression in stage 4S NB patients and clinical outcome. Our observation that nuclear E2F3 protein levels were elevated in primary tissues from stage $4 \mathrm{~S}$ NB relapsed or progressed compared with tumors from stage $4 S$ patients without any events, suggests that there may be continuous selection in the former tumors for unknown mechanisms that promote E2F3 activity. E2F3 may be subjected to further mechanisms of post-translation deregulation that are altered by oncogenesis. DNA methylation serves as a regulatory mechanism for transcription factor activity; therefore, we suggest that hypomethylation or common polymorphisms with enhancer activity influencing expression of protein coding gene at 6p22.3 might be the causes for the high expression of E2F3, but more studies need to be done. We postulate that $6 \mathrm{p} 22.3$ region becomes hypomethylated or that $E 2 F 3$ can be a susceptibility gene influenced by polymorphisms, lying within putative regulatory region, resulting in augmented E2F3 expression associated with disease phenotype and NB $4 S$ patient's outcome. Therefore, augmented E2F3 expression coming from an inherited polymorphism would impact neural crest cellular lineage commitment and predispose these cells to undergo malignant transformation. Additional studies are needed to develop more specific information about DNA methylation, histone modification, chromatin remodeling, or functional polymorphisms affecting gene expression during regression and differentiation of NB 4 S tumors.

\section{Materials and Methods}

\subsection{R2 Genomic Analysis and Visualization Platform}

We analyzed the data of RNA microarray experiments from three independent datasets consisting of 134 NB 4 S samples for RB1, TERT, E2F1, E2F2, E2F3 gene expressions extracted from the R2 Genomics Analysis and Visualization Platform (http://r2.amc.nl, last downloading: December 20, 2019). Selection criteria included at least 10 tumour samples of Stage 4S Neuroblastoma, no MYCN amplification, availability of time-to-event information for survival analysis. Three R2 databases fulfilling the inclusion criteria were identified, namely, Kocak-649 [26], Oberthuer-251 [27], and SEQC-RPM [28]. Furthermore, the data set SEQC-custom, which included the same samples of SEQC-RPM profiled by a different platform was also considered and analyzed separately.

\subsection{Immunofluorescence Analysis}

Immunofluorescence analysis was performed on formalin-fixed, paraffin-embedded NB specimens (4 $\mu \mathrm{m}$-thick) as previously described [42]. We used the mouse monoclonal antibody E2F-3 (D-2) (Santa Cruz Biotechnology, Dallas, TX, USA) and the CD56 (NCAM) polyclonal antibody (ThermoFisher Scientific, Waltham, MA, USA). We used isotype matched non-binding mAbs in all antibody staining experiments to avoid nonspecific reactivity. Results were photographically documented using fluorescence microscope Axio Imager M2 equipped with ApoTome System (Carl Zeiss, Oberkochen, Germany). For NB specimens, each tumor area tested contained malignant cells as assessed by histologic examination. Tumor cells were identified in each sample using the NB specific marker NCAM (CD56) [43] (Figure 3D,E). Quantification of immunofluorescence positive tumor cells was performed on serial tissue sections, thus allowing quantification in tumor areas selected by the pathologist. The proportion of immunofluorescence positive cells counted was at least 100 to 1000 cells and reported as percentage for the subsequent statistical analysis. 


\subsection{Patients and Tumor Samples}

We recovered a multi-institution retrospective series of primary NB tissue sections from 38 patients with stage 4S disease, diagnosed in the period from December 2000 to October 2011 in 27 centers of the Italian Association of Pediatric Hematology and Oncology (AIEOP) [44]. The tumors tissue specimens are stored in the BIT-NB Biobank of IstitutoGianninaGaslini, Genova, Italy. The patient data derived from Italian Neuroblastoma Registry (INBR) of AIEOP. The clinical characteristics of NB patients are collected in pseudo-anonymized manner and stored in a secure system at the Epidemiology and Biostatistics Unit of the IstitutoGianninaGaslini. This prospective Study Registry is conducted according to the principles of the Declaration of Helsinki (59th WMA General Assembly, Seoul, October 2008) and all its revisions, pertinent national laws and regulations, as well as the International Conference on Harmonization's Good Clinical Practice, taking into account the Directive 2001/20/EC of the European Parliament and of the Council April 2001. Medical records were abstracted at each institution, and clinical data including age at diagnosis, sex, stage, MYCN status, DNA index, histology, and outcome were collected. The patients were staged according to the International Neuroblastoma Staging System [6]. This study was conducted in accordance with the Declaration of Helsinki and approved by the Italian Institutional Ethics Committee (Measure $n^{\circ} 270 / 17$ related to the clinical study protocol IGG-NCA-AP-2016). Written informed consent was obtained from all patients or their legal guardians.

\subsection{Statistical Methods}

Event Free survival (EFS) was evaluated by the Kaplan-Meier method, splitting the NB patients into two groups on the basis of the median value of each gene expression profile. A sensitivity analysis was also carried out using two different cut-offs (the first and the last tertile of the gene expression distribution). The corresponding Hazard Ratios (HRs) were estimated by Cox regression model, applying the Firth's correction, based on a penalized likelihood function, in the presence of zero observed events in either one group [45]. For each considered gene, a meta-analytic common estimate of HR (mHR) and its related 95\% Confidence Intervals (95\% CI) were obtained using the random effect model by DerSimmonianandLeird (1986) [46], under the assumption of a log-normal distribution for both HR and mHR [47]. Survival analyses were also repeated by replacing the SEQC-RPM database with its homologous SEQC-custom, in order to check for the consistency of mHR estimates.

All analyses were performed by R statistical software, release 3.3.3 [48]. In detail, the R library survival was used to fit Cox regression models and to draw the corresponding Kaplan-Meier curves. The coxphf R library was employed to apply the Firth correction. Finally, an ad hoc routine in R language was implemented to estimate $\mathrm{mHR}$ and its related $95 \% \mathrm{CI}$.

\section{Conclusions}

In summary, interrogating in silicopublic NB databases, we found that high E2F3 expression level strongly correlates with worse outcome of NB patients with stage $4 \mathrm{~S}$ disease. Moreover, we screened primary tumor tissue from stage $4 \mathrm{~S} \mathrm{NB}$ for E2F3 protein expression using immunofluorescence, and we found that high level of nuclear E2F3 expression was strongly associated with disease relapse or progression.

Our results indicate that high expression of E2F3 can promote NB progression. The potential prognostic role of E2F3 in Stage 4S NB progression should be elucidated by extensive research.

Supplementary Materials: The following are available online at http://www.mdpi.com/2075-4418/10/5/315/s1.

Author Contributions: Conceptualization, A.P. and S.P.; methodology, S.P. and A.P.; statistical analysis, S.P.; resources, A.P.; data curation, S.P. and A.P.; writing — original draft preparation, S.P. and A.P.; writing-review and editing, S.P., M.O., R.H., and A.P.; supervision, S.P.; project administration, A.P.; funding acquisition, A.P. All authors have read and agreed to the published version of the manuscript. 
Funding: This research was funded by the Compagnia San Paolo; Cinque per mille dell'IRPEF Finanziamento della ricerca sanitaria; Finanziamento Ricerca Corrente, Ministero della Salute; Fondazione Maria Piaggio Casarsa; and Fondazione Italiana Neuroblastoma.

Acknowledgments: We thank Angela Rita Sementa (Laboratorio di Anatomia Patologica, IRCCS Istituto Giannina Gaslini, Genova) for providing the primary NB tumor specimens.

Conflicts of Interest: The authors declare no conflict of interest. The funders had no role in the design of the study; in the collection, analyses, or interpretation of data; in the writing of the manuscript; or in the decision to publish the results.

$\begin{array}{ll}\text { Abbreviations } \\ \text { NB } & \text { Neuroblastoma } \\ \text { TERT } & \text { telomerase reverse transcriptase } \\ \text { RB1 } & \text { retinoblastoma tumor suppressor } \\ \text { EFS } & \text { event-free survival } \\ \text { HR } & \text { hazard ratio } \\ \text { mHR } & \text { meta-analytic HR } \\ \text { ALT } & \text { alternative lengthening of telomeres } \\ \text { MYCN } & \text { v-mycmyelocytomatosis viral related oncogene neuroblastoma derived } \\ \text { GWAS } & \text { genome-wide association studies } \\ \text { INSS } & \text { International Neuroblastoma Staging System } \\ \text { INBR } & \text { Italian Neuroblastoma Registry } \\ \text { SIOPEN } & \text { International Society of Paediatric Oncology European Neuroblastoma } \\ \text { AIEOP } & \text { Italian Association of Pediatric Hematology and Oncology }\end{array}$

\section{References}

1. Matthay, K.K.; Maris, J.M.; Schleiermacher, G.; Nakagawara, A.; Mackall, C.L.; Diller, L.; Weiss, W.A. Neuroblastoma. Nat. Rev. Dis. Primers 2016, 2, 16078. [CrossRef]

2. Cheung, N.K.; Dyer, M.A. Neuroblastoma: Developmental biology, cancer genomics and immunotherapy. Nat. Rev. Cancer 2013, 13, 397-411. [CrossRef]

3. Johnsen, J.I.; Dyberg, C.; Wickström, M. Neuroblastoma-A Neural Crest Derived Embryonal Malignancy. Front. Mol. Neurosci. 2019, 12, 9. [CrossRef]

4. Speleman, F.; Park, J.R.; Henderson, T.O. Neuroblastoma: A Tough Nut to Crack. Am. Soc. Clin. Oncol. Educ. Book 2016, 35, e548-e557. [CrossRef] [PubMed]

5. Evans, A.E.; D'Angio, G.J.; Randolph, J. A proposed staging for children with neuroblastoma. Children's cancer study group A. Cancer 1971, 27, 374-378. [CrossRef]

6. Brodeur, G.M.; Pritchard, J.; Berthold, F.; Carlsen, N.L.; Castel, V.; Castelberry, R.P.; De Bernardi, B.; Evans, A.E.; Favrot, M.; Hedborg, F.; et al. Revisions of the international criteria for neuroblastoma diagnosis, staging, and response to treatment. J. Clin. Oncol. 1993, 11, 1466-1477. [CrossRef] [PubMed]

7. Haas, D.; Ablin, A.R.; Miller, C.; Zoger, S.; Matthay, K.K. Complete pathologic maturation and regression of stage IVS neuroblastoma without treatment. Cancer 1988, 62, 818-825. [CrossRef]

8. Tas, M.L.; Nagtegaal, N.; Kraal, K.C.J.M.; Tytgat, G.A.M.; Abeling, N.G.G.; Koster, J.; Pluijm, S.M.F.; Zwaan, C.M.; Keizer, B.; Molenaar, J.J.; et al. Neuroblastoma stage 4S: Tumor regression rate and risk factors of progressive disease. Pediatr. Blood Cancer 2019, 67, e28061. [CrossRef]

9. Nickerson, H.J.; Matthay, K.K.; Seeger, R.C.; Brodeur, G.M.; Shimada, H.; Perez, C.; Atkinson, J.B.; Selch, M.; Gerbing, R.B.; Stram, D.O.; et al. Favorable biology and outcome of stage IV-S neuroblastoma with supportive care or minimal therapy: A Children's Cancer Group study. J. Clin. Oncol. 2000, 18, 477-486. [CrossRef]

10. Taggart, D.R.; London, W.B.; Schmidt, M.L.; DuBois, S.G.; Monclair, T.F.; Nakagawara, A.; De Bernardi, B.; Ambros, P.F.; Pearson, A.D.; Cohn, S.L.; et al. Prognostic value of the stage 4S metastatic pattern and tumor biology in patients with metastatic neuroblastoma diagnosed between birth and 18 months of age. J. Clin. Oncol. 2011, 29, 4358-4364. [CrossRef] 
11. Twist, C.J.; Naranjo, A.; Schmidt, M.L.; Tenney, S.C.; Cohn, S.L.; Meany, H.J.; Mattei, P.; Adkins, E.S.; Shimada, H.; London, W.B.; et al. Defining Risk Factors for Chemotherapeutic Intervention in Infants with Stage4SNeuroblastoma: A Report from Children's Oncology Group Study ANBL0531. J. Clin. Oncol. 2019, 37, 115-124. [CrossRef] [PubMed]

12. Lavarino, C.; Cheung, N.K.; Garcia, I.; Domenech, G.; de Torres, C.; Alaminos, M.; Rios, J.; Gerald, W.L.; Kushner, B.; LaQuaglia, M.; et al. Specific gene expression profiles and chromosomal abnormalities are associated with infant disseminated neuroblastoma. BMC Cancer 2009, 9, 44. [CrossRef] [PubMed]

13. Decock, A.; Ongenaert, M.; De Wilde, B.; Brichard, B.; Noguera, R.; Speleman, F.; Vandesompele, J. Stage $4 S$ neuroblastoma tumors show a characteristic DNA methylation portrait. Epigenetics 2016, 11, 761-771. [CrossRef] [PubMed]

14. Brodeur, G.M. Spontaneousregression of neuroblastoma. Cell Tissue Res. 2018, 372, 277-286. [CrossRef] [PubMed]

15. Brodeur, G.M.; Bagatell, R. Mechanisms of neuroblastomaregression. Nat. Rev. Clin. Oncol. 2014, 11, 704-713. [CrossRef] [PubMed]

16. Alonso, M.M.; Fueyo, J.; Shay, J.W.; Aldape, K.D.; Jiang, H.; Lee, O.H.; Johnson, D.G.; Xu, J.; Kondo, Y.; Kanzawa, T.; et al. Expression of transcription factor E2F1 and telomerase in glioblastomas: Mechanistic linkage and prognostic significance. J. Natl. Cancer Inst. 2005, 97, 1589-1600. [CrossRef]

17. Vélez-Cruz, R.; Johnson, D.G. The Retinoblastoma (RB) Tumor Suppressor: Pushing Back against Genome Instability on Multiple Fronts. Int. J. Mol. Sci. 2017, 18, 1776. [CrossRef]

18. Pezzolo, A.; Pistorio, A.; Gambini, C.; Haupt, R.; Ferraro, M.; Erminio, G.; De Bernardi, B.; Garaventa, A.; Pistoia, V. Intratumoral diversity of telomere length in individual neuroblastoma tumors. Oncotarget 2015, 6, 7493-7503. [CrossRef]

19. Talluri, S.; Dick, F.A. Regulation of transcription and chromatin structure by pRB: Here, there and everywhere. Cell Cycle 2012, 11, 3189-3198. [CrossRef]

20. Morris, E.J.; Dyson, N.J. Retinoblastoma protein partners. Adv. Cancer Res. 2001, 82, 1-54.

21. Strieder, V.; Lutz, W. E2F proteins regulate MYCN expression in neuroblastomas. Biol. Chem. 2003, 278, 2983-2989. [CrossRef] [PubMed]

22. Olsen, R.R.; Otero, J.H.; Garcia-Lopez, J.; Wallace, K.; Finkelstein, D.; Rehg, J.E.; Yin, Z.; Wang, Y.D.; Freeman, K.W. MYCN induces neuroblastoma in primary neural crest cells. Oncogene 2017, 36, 5075-5082. [CrossRef] [PubMed]

23. Degregori, J.; Johnson, D.G. Distinct and overlapping roles for E2F family members in transcription, proliferation and apoptosis. Curr. Mol. Med. 2006, 6, 739-748. [CrossRef] [PubMed]

24. McNair, C.; Xu, K.; Mandigo, A.C.; Benelli, M.; Leiby, B.; Rodrigues, D.; Lindberg, J.; Gronberg, H.; Crespo, M.; De Laere, B.; et al. Differential impact of RB status on E2F1 reprogramming in human cancer. J. Clin. Investig. 2018, 128, 341-358. [CrossRef] [PubMed]

25. Welch, C.; Chen, Y.; Stallings, R.L. MicroRNA-34a functions as a potential tumor suppressor by inducing apoptosis in neuroblastoma cells. Oncogene 2007, 26, 5017-5022. [CrossRef] [PubMed]

26. Kocak, H.; Ackermann, S.; Hero, B.; Kahlert, Y.; Oberthuer, A.; Juraeva, D.; Roels, F.; Theissen, J.; Westermann, F.; Deubzer, H.; et al. Hox-C9 activates the intrinsic pathway of apoptosis and is associated with spontaneous regression in neuroblastoma. Cell Death Dis. 2013, 4, e586. [CrossRef] [PubMed]

27. Oberthuer, A.; Berthold, F.; Warnat, P.; Hero, B.; Kahlert, Y.; Spitz, R.; Ernestus, K.; König, R.; Haas, S.; Eils, R.; et al. Customized oligonucleotide microarray gene expression-based classification of neuroblastoma patients outperforms current clinical risk stratification. J. Clin. Oncol. 2006, 24, 5070-5078. [CrossRef]

28. Zhang, W.; Yu, Y.; Hertwig, F.; Thierry-Mieg, J.; Thierry-Mieg, D.; Wang, J.; Furlanello, C.; Devanarayan, V.; Cheng, J.; Deng, Y.; et al. Comparison of RNA-seq and microarray-based models for clinical endpoint prediction. Genome Biol. 2015, 16, 133. [CrossRef]

29. Brinkschmidt, C.; Poremba, C.; Christiansen, H.; Simon, R.; Schäfer, K.L.; Terpe, H.J.; Lampert, F.; Boecker, W.; Dockhorn-Dworniczak, B. Comparative genomic hybridization and telomerase activity analysis identify 2 biologically different groups of $4 \mathrm{~s}$ neuroblastomas. Br. J. Cancer 1998, 77, 2223-2229. [CrossRef]

30. Ackermann, S.; Cartolano, M.; Hero, B.; Welte, A.; Kahlert, Y.; Roderwieser, A.; Bartenhagen, C.; Walter, E.; Gecht, J.; Kerschke, L.; et al. A mechanistic classification of clinical phenotypes in neuroblastoma. Science 2018, 362, 1165-1170. [CrossRef]

31. Ginsberg, D. E2F3-a novel repressor of the ARF/p53 pathway. Dev. Cell 2004, 6, 742-743. [CrossRef] [PubMed] 
32. Miles, W.O.; Tschöp, K.; Herr, A.; Ji, J.Y.; Dyson, N.J. Pumilio facilitates miRNA regulation of the E2F3 oncogene. Genes Dev. 2012, 26, 356-368. [CrossRef] [PubMed]

33. Gamper, I.; Burkhart, D.L.; Bywater, M.J.; Garcia, D.; Wilson, C.H.; Kreuzaler, P.A.; Arends, M.J.; Zheng, Y.W.; Perfetto, A.; Littlewood, T.D.; et al. Determination of the physiological and pathological roles of E2F3 in adult tissues. Sci. Rep. 2017, 7, 9932. [CrossRef] [PubMed]

34. Tordai, A.; Wang, J.; Andre, F.; Liedtke, C.; Yan, K.; Sotiriou, C.; Hortobagyi, G.N.; Symmans, W.F.; Pusztai, L. Evaluation of biological pathways involved in chemotherapy response in breast cancer. Breast Cancer Res. 2008, 10, R37. [CrossRef]

35. Olsson, A.Y.; Feber, A.; Edwards, S.; TePoele, R.; Giddings, I.; Merson, S.; Cooper, C.S. Role of E2F3 expression in modulating cellular proliferation rate in human bladder and prostate cancer cells. Oncogene 2007, 26, 1028-1037. [CrossRef]

36. Feber, A.; Clark, J.; Goodwin, G.; Dodson, A.R.; Smith, P.H.; Fletcher, A.; Edwards, S.; Flohr, P.; Falconer, A.; Roe, T.; et al. Amplification and overexpression of E2F3 in human bladder cancer. Oncogene 2004, 23, 1627-1630. [CrossRef]

37. Cooper, C.S.; Nicholson, A.G.; Foster, C.; Dodson, A.; Edwards, S.; Fletcher, A.; Roe, T.; Clark, J.; Joshi, A.; Norman, A.; et al. Nuclear overexpression of the E2F3 transcription factor in human lung cancer. Lung Cancer 2006, 54, 155-162. [CrossRef]

38. Bosse, K.R.; Maris, J.M. Advances in the translational genomics of neuroblastoma: From improving risk stratification and revealing novel biology to identifying actionable genomic alterations. Cancer 2016, 122, 20-33. [CrossRef]

39. Maris, J.M.; Mosse, Y.P.; Bradfield, J.P.; Hou, C.; Monni, S.; Scott, R.H.; Asgharzadeh, S.; Attiyeh, E.F.; Diskin, S.J.; Laudenslager, M.; et al. A genome-wide association study identifies a susceptibility locus to clinically aggressive neuroblastoma at 6p22. N. Engl. J. Med. 2008, 358, 2585-2593. [CrossRef]

40. Russell, M.R.; Penikis, A.; Oldridge, D.A.; Alvarez-Dominguez, J.R.; McDaniel, L.; Diamond, M.; Padovan, O.; Raman, P.; Li, Y.; Wei, J.S.; et al. CASC15-S Is a Tumor Suppressor lncRNA at the 6p22 Neuroblastoma Susceptibility Locus. Cancer Res. 2015, 75, 3155-3166. [CrossRef]

41. Hurst, C.D.; Tomlinson, D.C.; Williams, S.V.; Platt, F.M.; Knowles, M.A. Inactivation of the Rb pathway and overexpression of both isoforms of E2F3 are obligate events in bladder tumours with 6p22 amplification. Oncogene 2008, 27, 2716-2727. [CrossRef]

42. Ognibene, M.; Podestà, M.; Garaventa, A.; Pezzolo, A. Role of GOLPH3 and TPX2 in Neuroblastoma DNA Damage Response and Cell Resistance to Chemotherapy. Int. J. Mol. Sci. 2019, 20, 4764. [CrossRef]

43. Ferreira-Facio, C.S.; Milito, C.; Botafogo, V.; Fontana, M.; Thiago, L.S.; Oliveira, E.; da Rocha-Filho, A.S.; Werneck, F.; Forny, D.N.; Dekermacher, S.; et al. Contribution of multiparameter flow cytometryimmunophenotyping to the diagnostic screening and classification of pediatric cancer. PLOS ONE 2013, 8, e55534. [CrossRef]

44. De Bernardi, B.; Di Cataldo, A.; Garaventa, A.; Massirio, P.; Viscardi, E.; Podda, M.G.; Castellano, A.; D'Angelo, P.; Tirtei, E.; Melchionda, F.; et al. Stage4sneuroblastoma: Features, management and outcome of 268 cases from the Italian Neuroblastoma Registry. Ital. J. Pediatr. 2019, 45, 8. [CrossRef] [PubMed]

45. Firth, D. Bias reduction of maximum likelihood estimates. Biometrika 1993, 80, 27-38. [CrossRef]

46. Der Simonian, R.; Laird, N. Meta-analysis in clinical trials. Control. Clin. Trials 1986, 7, 177-188. [CrossRef]

47. Tierney, J.F.; Stewart, L.A.; Ghersi, D.; Burdett, S.; Sydes, M.R. Practical methods for incorporating summary time-to-event data into meta-analysis. Trials 2007, 8, 16. [CrossRef] [PubMed]

48. R Core Team. R: A Language and Environment for Statistical Computing; R Foundation for Statistical Computing: Vienna, Austria, 2015.

(C) 2020 by the authors. Licensee MDPI, Basel, Switzerland. This article is an open access article distributed under the terms and conditions of the Creative Commons Attribution (CC BY) license (http://creativecommons.org/licenses/by/4.0/). 